\title{
Análisis del valor y su influencia en el rediseño y la sustitución de componentes en las mypes del sector textil de confecciones en la región Arequipa ${ }^{1}$ \\ Analysis of the value and its influence on the redesign and replacement of components in the mypes of the textile apparel sector in the Arequipa region.
}

\author{
Bernardo Ramón Dante De la Gala Velásquez, \\ Universidad Nacional San Agustín de Arequipa \\ bdelagala@unsa.edu.pe \\ Patricia Pilar Zirena Bejarano \\ Universidad Nacional San Agustín de Arequipa \\ pzirena@unsa.edu.pe \\ Angela Yuliana Arredondo Salas \\ Universidad Andina Néstor Cáceres Velásquez \\ jarredondo3@hotmail.com
}

El análisis del valor, el rediseño de productos y la sustitución de componentes son actividades claves en el desempeño de las microempresas del sector textil arequipeño; sin embargo, en la práctica, no son utilizadas en forma sistémica, realizándose con demoras en el tiempo de ejecución y deficiencias en los canales de comunicación, motivación y creatividad, entre otros, lo que hace que no sean aprovechadas adecuadamente (Chen \& Su, 2017; Aminoroaya Yamini, 2016). Un paso previo fundamental para realizar el rediseño de productos y/o la sustitución de componentes es el análisis del valor, según la propuesta de Meeker y McWilliams (2011); así, si los empresarios textiles lo aplicaran de acuerdo a lo que plantea el enfoque de análisis del valor, se podría mejorar la competitividad empresarial del sector.

El propósito de la investigación es demostrar la influencia que tiene el análisis del valor en la utilización del rediseño de productos y la sustitución de componentes, procesos muy usados en este tipo de empresas como parte de su quehacer productivo. Respecto a la metodología aplicada, se realizó un muestreo por conveniencia - empleando un cuestionario de medición tipo escala de Likert a 157 microempresarios de la región de Arequipa-, con el cual se obtuvo un nivel de confiabilidad de $\mathbf{0 . 8 3}$. El estudio determinó un nivel alto de utilización, de por

1. Los autores agradecen el apoyo de la Universidad Nacional San Agustín de Arequipa. También agradecemos los importantes aportes y comentarios de los árbitros y editores de $360^{\circ}$ Revista de Ciencias de la Gestión, de la Pontificia Universidad Católica del Perú, con los cuales se mejoró la calidad del presente estudio. 
encima del $63 \%$, en el análisis del valor, mientras que el rediseño de productos y la sustitución de componentes registró una utilización media de $\mathbf{5 2 . 9 0 \%}$. Adicionalmente, se identificó una influencia del análisis del valor sobre el rediseño de productos y la sustitución de componentes, en ambos casos con resultados altos encontrados mediante el sistema de ecuaciones estructurales (SEM); y también se determinó que no existe mediación del rediseño de productos o la sustitución de componentes alternadamente.

\section{Palabras clave: análisis del valor, rediseño de productos, sustitución de componentes, costos}

The value analysis, redesign and replacement of components are key activities in the performance of microenterprises in the Arequipa textile sector; however, in practice, they are not carried out in a systemic way, taking place with delays in execution time and deficiencies in the communication channels, motivation and creativity, among others, which means that they are not used properly (Chen \& Su, 2017; Aminoroaya Yamini, 2016). A preliminary step to redesign and/or replace components is the analysis of value, according to the proposal of Meeker and McWilliams (2011); that way, if textile entrepreneurs apply it according to what the value analysis approach raises, the business competitiveness of the sector could be improved.

The purpose of this research is to demonstrate the influence that value analysis has on the use of redesign and component replacement techniques, widely utilized to the productive work of this type of companies. Regarding the methodology that was applied, it should be mentioned that a sampling was carried out for convenience — using Likert scale measurement questionnaire to 157 microentrepreneurs in the Arequipa region-, and that a reliability level of 0.83 was obtained. The study determined a high level of use of these techniques - above $63 \%$ - in the analysis of the value, while the redesign and replacement of components registered an average use of $52.90 \%$. Additionally, an influence of the value analysis on the redesign and replacement of components was identified, in both cases with high results found through the system of structural equations (SEM); and it was also determined that there is no mediation of the redesign or replacement of components in turn. 


\section{Introducción}

Las empresas de confecciones textiles en el Perú representan el $16.30 \%$ del total de las mypes manufactureras, de las cuales 1157, aproximadamente, se encuentran en la región Arequipa, según datos internos proporcionados por la Gerencia Regional de la Producción al año 2018.

Arequipa es la segunda ciudad más industrializada del Perú cuando hablamos de actividad textil, la cual está fundamentalmente enfocada en la fabricación textil de fibra de alpaca y algodón. Por ello, y dada la problemática del sector — que basa su competitividad en el liderazgo de costos-, este estudio reviste especial importancia.

En este sector, donde predominan las empresas mypes, se fabrican productos para el mercado local, nacional e internacional, contribuyendo en gran medida a la producción para empresas exportadoras alpaqueras a través de tercerización. Estas grandes empresas son reconocidas en el mercado mundial por su alta especialización en el manejo de fibras de alpaca, contándose entre las más importantes de dicho sector productivo al Grupo Inca, Michell \& Cía., Franky \& Ricky, y Art Atlas, entre otras.

Para la presente investigación se realizó una revisión de la literatura disponible, encontrando estudios que parten de la teoría clásica de análisis del valor (Miles, 1962) —la cual, con el aporte de otros autores, evolucionó a ingeniería del valor-, entre ellos el de Meeker y McWilliams (2011), autores que proponen como sus técnicas el rediseño de productos y la sustitución de componentes, entre otras. Estas, a su vez, se identifican como las bases teóricas de la presente investigación, puesto que muestran características aplicables en el contexto de los microempresarios de la región. Por ello, propone el análisis del valor como una metodología que permite mejorar las condiciones de competitividad y comprende la identificación de las necesidades de los clientes, así como la asociación con la funcionalidad de cada producto, elemento y material que pudiera proponer un rediseño de productos o la sustitución de componentes a fin de mejorar el valor de los productos.

Las microempresas estudiadas despliegan un conjunto de actividades de análisis del valor, así como de rediseño de productos y sustitución de componentes, pero lo hacen de forma aislada, intuitiva y empírica, y no como elementos que forman parte de un proceso. La presente investigación da un aporte al conocimiento sobre cómo el análisis del valor influencia en el rediseño de productos y en la sustitución de componentes, y, a partir de ello, permite generar estrategias de uso del análisis del valor como paso previo al empleo del rediseño de productos y/o la sustitución de componentes en forma eficiente.

El estudio es de carácter cuantitativo no experimental y en su elaboración se utilizó estadística descriptiva y modelamiento de ecuaciones estructurales mediante programas estadísticos como el SPSS y AMOS. El análisis de mediación, a su vez, fue realizado utilizando la macro Process de Hayes (2017).

La investigación se ha estructurado en los siguientes apartados: el marco teórico, la metodología cuantitativa de diseño transversal, el análisis y la discusión de resultados, las conclusiones y limitaciones del estudio, y las líneas futuras de investigación. 


\section{Marco teórico}

Con el propósito de comprender conceptualmente la forma en que los microempresarios de la región de Arequipa incrementan el valor en sus productos y servicios, se realizó una revisión de literatura por medio de textos físicos y virtuales, tomando en consideración palabras clave como "análisis del valor», «rediseño», "sustitución de componentes» y "costos». De esta forma, a través de diferentes bases de datos, se encontró información sobre la definición clásica de valor, como la propuesta de Garcia Melón et al. (2010), quienes afirman que el valor es la relación entre el grado de satisfacción de las necesidades y los recursos que se usan para conseguir esa satisfacción.

\subsection{Análisis del valor}

Se puede abordar el tema del análisis del valor a través del concepto aportado por Miles (1962), el cual surge al final de la Segunda Guerra Mundial e identificó una problemática radicada en la escasez de materiales. Lawrence Miles, directivo de la General Electric entonces, era el responsable de conseguir materiales alternativos para reemplazar la materia prima que no se conseguía y que había sido usada con regularidad en los procesos productivos. Así, él encuentra que otros materiales podían reemplazar adecuadamente a los usados comúnmente y, lo más importante, con menor costo, mejorando la estructura de costos de los productos terminados.

Más adelante, y a raíz de esta experiencia, se descubre que se podían agregar ciertos atributos al producto que no habían sido considerados hasta el momento, e inclusive se empiezan a tomar en cuenta aspectos relacionados con la estética y belleza, como el hecho de que no debe haber contradicción entre lo bello y lo útil, pues el objeto posee belleza desde el momento en que su forma es expresión manifiesta de su función (Souriau, 1998). Así, se adiciona el interés por la conveniencia al uso del producto, en función a los deseos y necesidades de los consumidores.

Con estos antecedentes, y aceptando que los productos deben ser ofrecidos al cliente con costos reducidos, se planteó el análisis del valor como alternativa y solución para ello. Después de eso, se agregó un concepto importante que consiste en ofrecer funciones adicionales al cliente a través del producto, de forma que el valor puede ser identificado y percibido por el consumidor a través de la minimización de los costos como consecuencia del análisis de las funciones involucradas (Miles, 1962). No obstante, el concepto tiene un aspecto altamente subjetivo, puesto que el valor apreciado por una persona no puede trasladarse idénticamente a otra (Chhabra \& Tripathi, 2014).

El propósito de realizar un análisis en la función de costos es mejorar el valor del producto a través de un trabajo serio y realizado en forma sistemática (Morales, 2002). Tomando en cuenta que es muy difícil mejorar el valor a través de técnicas convencionales puesto que estas consumen demasiado tiempo en el análisis de las funciones involucradas, y más aún dado que estas, hoy en día, son sumamente sofisticadas debido a la exigencia de los consumidores (Chan-Sik, Ho-Jum, Hee-Taek, Jong-Ho \& Akeem, 2017), se propone la aplicación del análisis del valor como una técnica novedosa que, aunque ha sido muy usada en el sector construcción —como muestra la evolución del 
modelo en países como Estados Unidos, Japón y Gran Bretaña, entre otros- y también, en menor medida, en campos como la fabricación de accesorios automotrices — donde se considera adicionalmente la investigación de mercado y la mejora de la calidad del producto (Rui et al., 2016; Bock \& Pütz, 2016)—, todavía no acumula experiencias en el sector de manufactura de microempresarios textiles.

El análisis del valor puede ser definido como la revisión planificada y ordenada en la que se examina la composición de los materiales de un producto, el diseño y la forma de producción, de modo que las modificaciones y mejoras que se pueden hacer puedan llevar a un nuevo diseño del producto que, de acuerdo a los antecedentes encontrados, se reconoce como rediseño, cumpliendo esencialmente con la condición de no reducir el valor del producto ni disminuir la calidad del mismo; sino, por el contrario, mejorar sus atributos (Abdullah, EYT \& Al-fadhli, 2015).

Por su naturaleza, el análisis del valor plantea un desafío interdisciplinario que busca mejorar el producto, y debe realizarse través de un análisis de funciones que identifique la aplicación sistémica de las técnicas reconocidas para identificar qué funciones pueden ser mejoradas, lo cual, sumado a un desempeño adecuado, busca que el producto alcance un menor costo (Mana, 2017). Hoy en día, esta herramienta se puede considerar un método creativo e innovador que busca satisfacer las necesidades de los usuarios a través de un conjunto de soluciones, teniendo en cuenta criterios funcionales (Houshang, Hassan, \& Abdolhossein, 2012). Así, se pueden identificar las siguientes etapas:

- Análisis de las necesidades de los usuarios: se debe evaluar la importancia de las necesidades para establecer prioridades entre sus demandas o necesidades, reconociendo que se debe recoger esa información, que será utilizada en el momento de plantear alternativas de diseño y rediseño del producto.

- Análisis de las funciones del producto: se deben definir las funciones del producto que ofrece la empresa para relacionarlas con las necesidades del consumidor.

- Análisis de los componentes del producto: se debe elaborar un listado de funciones y de componentes, identificando los costos en los que se incurrirá en cada uno de ellos (Garcia Melón et al., 2010).

Una vez ejecutadas las acciones propuestas en cada etapa, son analizadas y evaluadas con el fin de buscar la forma de mejorar el valor del producto. Para ello, se pueden proponer dos alternativas iniciales para alcanzar y mejorar el valor: el rediseño de productos y la sustitución de componentes, de acuerdo al modelo que tomamos como referencia.

Respecto a la primera alternativa, se puede mencionar que podría reducirse el costo del producto si se encuentra una forma o presentación nueva, lo que se identificará como rediseño. En el caso de la segunda alternativa, se pueden usar piezas de menor costo, puede disminuirse el número de piezas o pueden crearse nuevas funciones con pequeños cambios en el producto. No obstante, más allá de la ruta elegida, el rediseño de productos genera la mayor reducción de costos (Meeker \& McWilliams, 2011) y, por esa razón, vale la pena centrarnos en este tema en primera instancia. 


\subsection{Técnicas para reducir el costo del producto}

\subsubsection{Rediseño de productos}

Existen varias técnicas para alcanzar la reducción de costos, entre ellas el rediseño de productos y la sustitución de componentes (Meeker \& McWilliams, 2011).

Rediseñar un producto implica darle una nueva presentación, es decir, modificar las características y atributos que le permitan adquirir una apariencia relativa o totalmente diferente. Para ello, se definen los aspectos formales sobre presentación y forma en que se percibe el producto. La correcta definición de los atributos que se relacionan con el producto y su posterior implementación en él son de vital importancia para el éxito empresarial, teniendo en cuenta que dichas características se centran en dos aspectos (Guerrer, Hernandiz, \& Begoña, 2017).

En primer lugar están los relacionados con las personas, como los factores sensoriales, psicosociales y perceptivos que buscan la armonización e identidad entre el producto y el cumplimiento de las expectativas del consumidor (Lenau \& Boelskfte, 2004). La imagen del producto es percibida por el consumidor y es probablemente determinante en la decisión de compra, por lo que vale la pena poner interés en la presentación que será captada por el cliente.

En segundo lugar están los valores más "duros», asociados a los aspectos y características funcionales y de utilidad de un producto. Según Guerrero Valenzuela, Hernandis Ortuño y Agudo Vicent (2014), estos se definen a partir del cumplimiento de los objetivos y especificaciones prescritas por la empresa o por el cliente. Para nuestro estudio, es fundamental plantear alternativas concretas para realizar el proceso de rediseño de productos, que puede iniciarse con técnicas sumamente sencillas como el brainstorming hasta llegar a convertirse en una disciplina (ciencia del diseño) en la que la característica fundamental es que identifica un ambiente absolutamente artificial, construido por el ser humano aprovechando herramientas tecnológicas propias del entorno actual, en el que se pueden señalar como fundamentales el punto de partida — que vendría a ser el producto —, el objeto de análisis y la búsqueda del recorte de costos. Asimismo, se aplicarán procesos mentales que plasmen propuestas creativas y que se implementarán a través de talleres en los que participen trabajadores que puedan precisar las necesidades de los consumidores.

El diseño o rediseño del producto es resultado de la aplicación del conocimiento, la tecnología y la práctica profesional, y generalmente se aplica para resolver problemas de disminución de costos y de incremento de funciones, implicando una transformación creativa de la realidad existente (Gonzales, 2007). Para alcanzar dicho objetivo, se deben identificar las funciones primarias y secundarias, pues la metodología del análisis del valor indica que se debe describir cada componente en forma de verbo acompañado de un sustantivo que describa la función. De acuerdo a ello, se propone el análisis de funciones de la siguiente forma:

- Función relacionada al cliente: funciones que se espera cumpla el producto de acuerdo a los requerimientos y expectativas de los clientes.

- Función relacionada al producto: es aquella que se relaciona con los elementos 
que conforman el producto, los mismos que generarán la disminución de los costos: materiales, procesos, mano de obra y costos indirectos en los que se incurren.

Inmediatamente se hayan identificado las funciones, se puede realizar el análisis detallado de los elementos, lo que conduce a la mejora del valor, teniendo en cuenta que todo producto debe ser identificado y asociado a un verbo, de tal forma que el verbo identifica y describe la acción y el nombre del objeto indica sobre qué se aplica dicha acción.

Asimismo, se identifican las siguientes etapas por las que pasa el proceso:

- Primera etapa: inicialmente, hay que identificar la estructura del producto y las funciones a realizar, tomando en cuenta que las funciones son acciones de trabajo o cosas. También se analiza cada uno de sus componentes, de acuerdo a la función desempeñada. Inmediatamente, se debe pensar en cómo se podrían mejorar las funciones que cumple dicho producto; por ejemplo, cómo mejorar la apariencia, cómo extender la durabilidad o cómo mejorar la adaptabilidad a las necesidades del usuario. Así, toda mejora busca ofrecer mayor calidad y mejor aún si se logra a menor costo (Universidad Interamericana para el Desarrollo, s.f.).

- Segunda etapa: se debe identificar cada función —o los grupos de funciones —, descomponiendo los elementos que las componen y señalando además la importancia y el aporte al producto final, lo que nos acercará a la identificación del costo de cada una. Sobre esa base, se propone elaborar un cuadro resumen, separando las funciones primarias y secundarias para facilitar la ubicación de la función en la que se aplicará el cambio o la mejora.

- Tercera etapa: una vez identificada la función a mejorar, se deben plantear objetivos y metas que definirán los cambios a los que se pretende llegar. Generalmente, se inicia el estudio reconociendo el costo mayor en términos absolutos y porcentuales con el fin de enfocar esfuerzos en lo más importante.

- Cuarta etapa: se acopia información necesaria y confiable, construyendo bases de datos de proveedores, materiales alternativos y costos de cada elemento que forma parte del costo de producción. Esta información puede servir para pautear y diseñar procesos consecuentes.

- Quinta etapa: generar alternativas de mejora, de rediseño de productos o de sustitución de componentes, tomando en cuenta las tendencias y evolución de los gustos y requerimientos de los clientes, además de considerar la viabilidad de las propuestas presentadas.

- Sexta etapa: evaluar y seleccionar la mejor alternativa de acuerdo a los objetivos planteados y al valor que genera. Es importante que esto sea medido financieramente para asegurar el éxito del proceso.

De acuerdo a lo planteado anteriormente, se propone un feedback sobre lo conseguido a través del proceso, creando una historia sobre los procesos realizados y que podrían ser muy bien aprovechados en iniciativas futuras de rediseño de productos 
y de sustitución de componentes. El análisis del valor ayudará, por tanto, a modificar los productos, de manera que, sin dejar de cumplir la función con la misma eficacia, se obtengan a un menor costo, aumentando así su valor o mejorando la capacidad de realizar las funciones del producto, más que proporcionalmente sus costos (Centro Aragonés de Diseño Industrial, s.f.). Si este análisis se realizó con la seriedad del caso, se puede asegurar que los resultados serán sumamente beneficios para la empresa, reflejándose los mismos en la situación financiera y operativa al hacerla sumamente competitiva.

\subsubsection{Sustitución de componentes}

De acuerdo con Meeker y McWilliams (2011), la sustitución de componentes es el segundo método más utilizado para reducir costos, después del rediseño de productos, y consiste en reemplazar un componente utilizado en la fabricación por otro con un costo menor. Esta acción tiene relación directa con la estrategia de hacer y de compra. Hacer involucra la decisión de producir un ítem o una parte de él dentro de la organización, mientras que comprar implica la decisión de adquirir un producto o una parte de él fuera de la organización (externalización). Ambas decisiones son evaluadas usualmente mediante los costos, la calidad y la funcionabilidad de los productos y/o partes (Sosa Vásquez \& Chávez Alonso, 2014).

La externalización que implica la compra de productos, partes u otros recursos requeridos por las organizaciones también está relacionada con el conocimiento tecnológico de la fuerza laboral de las organizaciones; así, a mayor conocimiento, menor será la probabilidad de externalizar sus operaciones (Gupta \& Gupta, 1992; Poppo \& Zenger, 1998; Alvarez Suescun, 2007).

En ese sentido, Ramírez Gómez (2010) plantea que los costos de transacción significan ceder los derechos de propiedad sobre sus factores productivos, tomando en cuenta precios, calidad del producto, mano de obra y otros recursos productivos (Martínez Argüelles \& Rubiera Morollón, 2005); es por ello que en la sustitución de componentes es indispensable tener en cuenta lo antes mencionado. Además, el tomar la decisión de hacer o comprar tiene que ver con un conjunto de aspectos que se deben de analizar profundamente, pues de ello depende mejorar la productividad de los negocios sin sacrificar la rentabilidad, el nivel de calidad, la funcionabilidad y otros aspectos del producto en estudio.

Un primer aspecto es identificar si se tienen recursos ociosos en la organización (maquinaria, equipo, mano de obra), lo que implicaría la decisión de escoger utilizar estos recursos ociosos y, con ello, elevar la productividad (Polimeni, Adelberg \& Fabozzi, 1998). Un segundo aspecto a tomar en cuenta es la valoración de los costos; por ejemplo, fabricar los componentes internamente — dentro de la organización— por lo general representa costos menores que los de la adquisición de partes a proveedores. Asimismo, otro aspecto relevante a tomar en cuenta es el del "justo a tiempo», pues muchas empresas no cuentan con proveedores confiables en cuanto a la entrega oportuna de las partes que requieren para su fabricación y deciden por ello manufacturar sus propios componentes para garantizar un tiempo de entrega oportuno al ensamble de sus productos. 
La calidad es un factor crítico y estratégico a tener en cuenta en la decisión de hacer y comprar, y muchas veces los componentes que se ofertan en el mercado no guardan la calidad requerida para la fabricación de los productos de la empresa. Así, en resguardo del nivel de calidad aceptado por el mercado, muchas empresas se ven en la necesidad de manufacturar sus propios componentes. En esa misma línea, es posible que los componentes tengan un nivel de calidad aceptable, pero no cuenten con algún atributo, lo que disminuye la funcionabilidad del producto, escenario que también empuja a algunas empresas a producir sus propios componentes, aunque ese no sea el core de sus respectivos negocios.

De igual forma, la adaptabilidad y el alineamiento de recursos, entre otras capacidades dinámicas de orden superior, generan respuestas agiles y rápidas de las empresas hacia el mercado, mejorando su competitividad (Eckstein, Goellner, Blome \& Henke, 2015; De la Gala \& Arredondo, 2019). En este sentido, el empleo de las técnicas de rediseño y sustitución de componentes sustentadas en el análisis del valor debe de ejecutarse en forma sistémica, adaptando y alineando los recursos productivos para dar respuestas ágiles al mercado, contribuyendo de esta manera con la mejora de la productividad y, por ende, de la competitividad de los negocios.

De acuerdo a lo revisado en los puntos anteriores, podemos decir que no existen trabajos empíricos que hayan revisado las relaciones que se dan entre el análisis del valor, el rediseño de productos y la sustitución de componentes; por lo tanto, planteamos el siguiente modelo teórico (ver figura 1), donde se visualizan las dos primeras hipótesis de trabajo orientadas a confirmar las relaciones directas que se dan entre las variables antes mencionadas.

- Hipótesis 1: el análisis del valor tiene una relación directa con el rediseño de productos, la misma que influye positivamente en él.

- Hipótesis 2: el análisis del valor tiene una relación directa con la sustitución de componentes, la misma que influye positivamente en ella.

Figura 1. Modelo teórico 1: influencia del análisis del valor en el rediseño de productos y en la sustitución de componentes
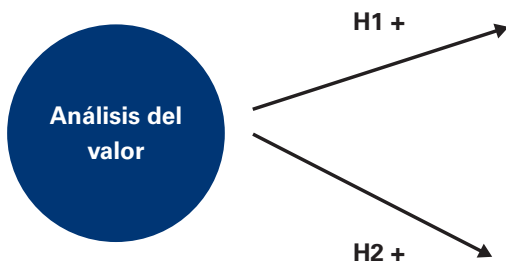

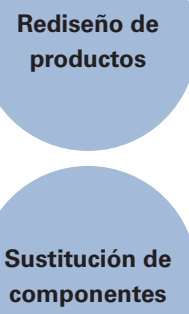

Rediseño de

componentes 
La figura 1 ilustra el modelo teórico 1, que plantea dos hipótesis de trabajo: la primera sobre la relación directa del análisis del valor con el rediseño de productos, y la segunda sobre la relación del análisis del valor y la sustitución de componentes. De igual manera, con el fin de determinar las relaciones indirectas que se podrían estar dando dentro de las variables de estudio, se han planteado dos hipótesis de trabajo adicionales:

- Hipótesis 3: el rediseño de productos tiene una relación indirecta de mediación entre el análisis del valor y la sustitución de componentes, la misma que influye positivamente en esta última.

- Hipótesis 4: la sustitución de componentes tiene una relación indirecta de mediación entre el análisis del valor y el rediseño de productos, la misma que influye positivamente en esta última.

La siguiente figura visualiza las hipótesis antes mencionadas:

Figura 2. Modelo teórico 2: mediación del rediseño de productos y/o sustitución de componentes

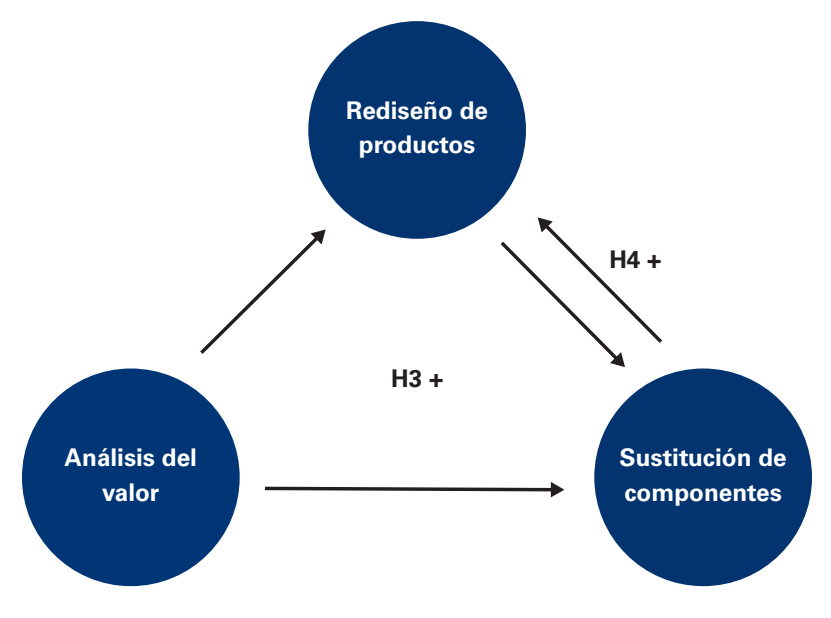

Fuente: elaboración propia.

La figura 2 ilustra el modelo teórico 2, donde se plantean dos hipótesis de trabajo de variables mediadoras: la primera mediación es la hipótesis 3 del rediseño de productos, mediando entre el análisis del valor y la sustitución de componentes; y la segunda mediación es la hipótesis 4, que plantea la mediación de la sustitución de componentes, mediando entre el análisis del valor y el rediseño de productos.

\section{Metodología}

\subsection{Población y muestra}

La presente investigación fue realizada en el año 2018, periodo en el cual se enviaron 300 encuestas a microempresarios del sector textil de confecciones en la región Arequipa. De 
ellos, solo 157 microempresarios aceptaron ser parte del estudio, Asimismo, cabe señalar que la población en estudio para este sector económico es de 1157 microempresarios formales, según estadística interna de la Gerencia de la Producción del Gobierno regional de Arequipa, Perú, al 2018.

Al inicio de las encuestas, los microempresarios recibieron un trabajo de sensibilización, resaltando la importancia de su participación y, sobre todo, la calidad en el detalle y la veracidad de la información que iban a proporcionar; también se les informó que en base a los resultados del estudio, se podrían plantear alternativas de mejora para la competitividad de las microempresas del sector, hecho que podría beneficiarlos directamente, lo que generó en ellos una alta expectativa.

En el estudio, se obtuvo una alta tasa de respuesta, que alcanzó el 13.56\%, cifra que resulta importante para el desarrollo de la presente investigación, dado que en investigaciones similares se registran tasas de respuesta entre 9 y $14 \%$, aproximadamente.

El primer objetivo propuesto fue determinar si existe influencia entre el análisis del valor y el rediseño de productos y/o sustitución de componentes. Con este objetivo en mente, se propuso encontrar las relaciones significativas que se dan entre las variables para demostrar la causalidad entre el análisis de valor y el rediseño de productos, así como entre dicho análisis y la sustitución de componentes, según el modelo teórico propuesto (ver figura 1). Este objetivo constituye la esencia de esta investigación.

El segundo objetivo fue identificar si el rediseño de productos y la sustitución de componentes se comportan como variables mediadoras entre el análisis del valor y cada una de ellas, respectivamente. Este objetivo tenía el propósito de demostrar si estas dos variables generaban sinergias en su empleo conjunto; en concreto, si el rediseño de productos mediaba entre el análisis del valor y la sustitución de componentes, por una parte, y si la sustitución de componentes mediaba entre el análisis del valor y el rediseño de productos, por la otra, siendo ambos modelos teóricos propuestos (ver figura 2).

Por último, se fijó como tercer objetivo precisar el nivel de utilización del análisis del valor, dado que este análisis previo es considerado por los microempresarios como fundamental en tanto sirve como base para la implementación del rediseño de productos y/o la sustitución de componentes, procesos con los cuales se debería generar competitividad en estos negocios. Además, se requiere precisar el nivel de utilización del rediseño de productos y/o la sustitución de componentes en la gestión de estos negocios, como lo sugiere la teoría y la lógica de los empresarios mypes contactados en la sesión de inducción comentada anteriormente.

\subsection{Diseño de investigación}

El presente estudio, de carácter exploratorio y empírico, tiene un enfoque cuantitativo de tipo no experimental, al no realizarse manipulación de variables, siendo su diseño de corte transversal, pues el recojo de la información se hizo en un solo periodo de tiempo. Es necesario comentar que estos estudios también son conocidos como estudios ex post facto, dado que el recojo de la información se realiza después de que ocurrieron los hechos; asimismo, tiene como propósito demostrar la influencia del análisis del valor en el rediseño de productos y la sustitución de componentes, alcanzando con esto un nivel explicativo. 


\subsection{Variables y escalas}

Para el levantamiento de la información se aplicó un cuestionario, con escalas de medición tipo Likert de cinco posiciones, el mismo que tiene catorce indicadores, herramienta con la que se recogió la información para las tres variables en estudio. A su vez, la primera de ellas, el análisis del valor, está constituida por las siguientes dimensiones: la primera es el establecimiento de la línea base, medida por tres indicadores; la segunda, el monitoreo de costos; la tercera, el análisis de costos; y la cuarta, la configuración de los productos, que son medidos —cada uno de ellos_ por un solo indicador, respectivamente. Estas dimensiones son fundamentales para el desarrollo de la investigación pues son los componentes de la variable causa, que se propone en este estudio.

La segunda variable de análisis es el rediseño de productos, para el cual se utilizaron cinco indicadores de medición; mientras que en el caso de la tercera variable, la sustitución de componentes, se utilizaron tres indicadores para su medición. A continuación mostramos la tabla 1, que resume lo recién mencionado.

Tabla 1. Variables, dimensiones, indicadores y escalas de medición

\begin{tabular}{|c|c|c|c|}
\hline Variables & Dimensiones & Indicadores & Medición \\
\hline \multirow{6}{*}{$\begin{array}{l}\text { Análisis } \\
\text { del valor }\end{array}$} & \multirow{3}{*}{ Línea base } & Tienen identificados los materiales utilizados por cada producto & Escala Likert 1-5 \\
\hline & & $\begin{array}{l}\text { Tienen identificadas las operaciones que se necesitan en la } \\
\text { fabricación de sus productos }\end{array}$ & Escala Likert 1-5 \\
\hline & & $\begin{array}{l}\text { Tienen identificados los gastos y materiales de mantenimiento } \\
\text { que se utilizan en la fabricación }\end{array}$ & Escala Likert 1-5 \\
\hline & $\begin{array}{l}\text { Monitoreo de } \\
\text { costos }\end{array}$ & $\begin{array}{l}\text { Tienen identificadas las cantidades de materiales, mano de } \\
\text { obra y gastos utilizados por cada producto }\end{array}$ & Escala Likert 1-5 \\
\hline & $\begin{array}{l}\text { Análisis de } \\
\text { costos }\end{array}$ & $\begin{array}{l}\text { Tienen identificadas los precios de materiales, mano de obra y } \\
\text { gastos utilizados por cada producto }\end{array}$ & Escala Likert 1-5 \\
\hline & Configuración & $\begin{array}{l}\text { Tienen identificados las configuraciones de los productos más } \\
\text { vendidos }\end{array}$ & Escala Likert 1-5 \\
\hline \multirow{5}{*}{\multicolumn{2}{|c|}{ Rediseño de productos }} & $\begin{array}{l}\text { Tienen identificados los procesos, subprocesos y operaciones } \\
\text { por cada producto }\end{array}$ & Escala Likert 1-5 \\
\hline & & $\begin{array}{l}\text { Usualmente genera alternativas de mejora de los procesos } \\
\text { productivos }\end{array}$ & Escala Likert 1-5 \\
\hline & & $\begin{array}{l}\text { Evalúa los costos de cada alternativa planteada para la mejora } \\
\text { de los procesos productivos }\end{array}$ & Escala Likert 1-5 \\
\hline & & $\begin{array}{l}\text { Genera planes de implementación de cada alternativa plantea- } \\
\text { da para la mejora de los procesos productivos }\end{array}$ & Escala Likert 1-5 \\
\hline & & $\begin{array}{l}\text { Gestiona el acuerdo y compromiso entre sus miembros colabora- } \\
\text { dores para llevar adelante la alternativa planteada }\end{array}$ & Escala Likert 1-5 \\
\hline \multirow{3}{*}{\multicolumn{2}{|c|}{$\begin{array}{l}\text { Sustitución de compo- } \\
\text { nentes }\end{array}$}} & $\begin{array}{l}\text { En el caso de que un insumo de una marca } X \text { que suelen } \\
\text { adquirir tenga un precio elevado, buscan algún insumo sustituto } \\
\text { de otra marca }\end{array}$ & Escala Likert 1-5 \\
\hline & & $\begin{array}{l}\text { Tienen identificados insumos o materiales que pueden ser } \\
\text { reemplazados por otros de menor coste y rendimiento, pero } \\
\text { que no alteran o afectan la calidad del producto final }\end{array}$ & Escala Likert 1-5 \\
\hline & & $\begin{array}{l}\text { Tienen identificados insumos o materiales que son de alto } \\
\text { rendimiento y alto costo, pero que a largo plazo son rentables }\end{array}$ & Escala Likert 1-5 \\
\hline
\end{tabular}




\subsection{Confiabilidad}

La confiabilidad del cuestionario fue medida mediante el Alfa de Cronbach, dando un resultado para los catorce indicadores planteados en la investigación de un alfa 0.83 , considerado muy superior al 0.70 aceptado por diversos autores (Carmines \& Zeller, 1979). Además, Fornell \& Larcker (1981) plantea que el nivel de aceptación debería ser de un 0.80. Este planteamiento es compartido por un conjunto de autores con enfoques más estrictos.

\subsection{Validez}

El análisis de la validez convergente y discriminante se realizó utilizando el coeficiente de correlación de Pearson, el mismo que mide el nivel de covariación entre los respectivos indicadores que corresponden a una determinada variable, correlacionándolos entre sí para el caso de la validez convergente y correlacionando los mismos indicadores con las otras variables para el caso de la validez discriminante (cross loading). Este análisis demostró altos índices de correlación de los indicadores con cada una de sus variables y menores índices de correlación de esos indicadores con las otras variables de estudio, demostrándose así la validez convergente y discriminante de los indicadores y sus variables.

Además, se utilizó el modelamiento mediante ecuaciones estructurales en el programa AMOS. En el modelo de medida, donde se realiza el análisis factorial confirmatorio de las variables observables (indicadores) y cada una de sus variables latentes (variables del estudio), se confirma lo mencionado anteriormente, ya que se observan índices de ajuste global (RMSEA, PCLOSE, CMIN/DF) buenos y aceptables (ver tabla 2). En el caso de los indicadores incrementales (TLI y CFI), estos superan el 0.90 como nivel de aceptación; y en el caso de los índices de parsimonia (PNFI y PCFI), se observan valores mayores a 0.50 en el nivel de aceptación, confirmándose con ello la validez discriminante y divergente de las variables de estudio y sus indicadores. En la tabla 2 se aprecia un resumen de los índices de los modelos de ecuaciones estructurales.

\subsection{Técnicas de análisis}

Para el análisis estadístico se han utilizado los programas SPSS 25 y AMOS 25, mientras que el análisis de confiabilidad se confirmó mediante el modelado de ecuaciones estructurales, utilizando máxima verosimilitud en su modelo de medida.

En el análisis del primer objetivo de la investigación se utilizó la técnica de ecuaciones estructurales, mediante máxima verosimilitud en su modelo de estructura, para determinar la influencia del análisis del valor en el rediseño y/o sustitución de componentes (ver figura 1).

En el caso del segundo objetivo de investigación, donde se trata de precisar si existe mediación entre la sustitución de componentes y/o el rediseño, y el análisis del valor (ver figura 2), se empleó la macro Process de Hayes (2017).

Para el tercer objetivo, precisar el nivel de utilización de las variables de estudio —análisis del valor, rediseño de productos y sustitución de componentes-, se utilizaron las técnicas estadísticas de análisis de límites superior e inferior, establecimiento de intervalos de clase, recodificación y frecuencias. 


\section{Resultados y discusión}

4.1. Influencia del análisis del valor en el rediseño de productos y/o la sustitución de componentes: contraste de hipótesis

Para alcanzar el primer objetivo de la investigación se utilizó el programa estadístico AMOS en el modelo de estructura, empleándose la técnica de estimación de máxima verosimilitud para determinar la influencia del análisis del valor en el rediseño de productos, así como en la sustitución de componentes, lo que confirmó el modelo teórico propuesto en la figura 1.

Asimismo, se muestran índices de ajuste global buenos con valores RMSEA de 0.055, PCLOSE de 0.351 y CMIN/DF de 1.479, todos ellos aceptables. En cuanto a los índices incrementales, tenemos un TLI y un CFI de 0.928 y 0.948 , respectivamente, ambos mayores a 0.90 y, por lo tanto, aceptables. Y, sobre los índices de parsimonia, se registran índices adecuados, mostrándose un PNFI de 0.625 y un PCFI de 0.689, los dos dentro de los niveles de aceptación, en el modelo de ecuaciones estructurales de estimadores no estandarizados. Esto se puede apreciar en la tabla 2, que muestra diversos índices de bondad de ajuste.

Adicionalmente, se realizó un análisis de correlación dentro del modelo AMOS, obteniéndose para la relación análisis del valor y rediseño de productos un coeficiente de 0.957 a un nivel de significación al 0.01, aceptándose la hipótesis H 1. Para la relación análisis del valor y sustitución de componentes se observa un coeficiente de correlación de 0.2678 a un nivel de significación de 0.043, aceptándose la hipótesis H 2 .

Es necesario precisar que en ambos casos los niveles de significancia son inferiores a un 0.05 y presentan un nivel de aprobación al 95\% de confianza; por lo tanto, se acepta una correlación significativa en ambas relaciones observadas en la figura 3.

Analizadas las relaciones de las dos variables, podemos apreciar que la mayor relación directa positiva se da entre el análisis del valor en relación directa positiva con el rediseño de productos, con un coeficiente de correlación de 0.957. De igual manera, la sustitución de componentes tiene un nivel de relación bajo, con un coeficiente de correlación de 0.2678, como se puede contemplar en la figura 3.

Figura 3. Influencia del análisis del valor en el rediseño de productos y en la sustitución de componentes

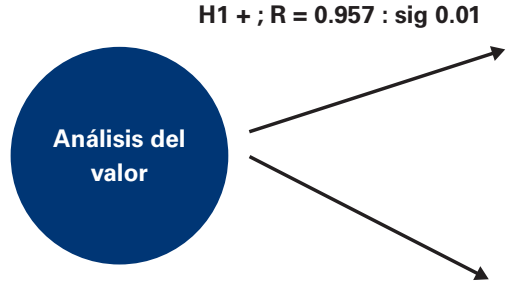

$\mathrm{H} 2+; \mathrm{R}=0.2678: \operatorname{sig} 0.043$

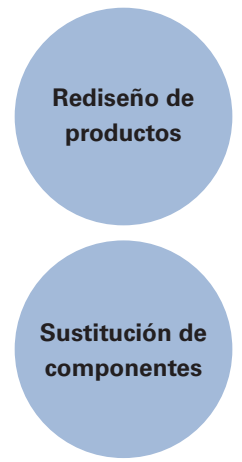

Fuente: elaboración propia 
La figura 3 muestra los resultados del modelo 1, donde se observan los coeficientes de correlación, así como su nivel de significancia en ambos casos aceptados.

Es necesario comentar que en el uso de ecuaciones estructurales (SEM-COB), especialmente el basado en covarianzas —como el AMOS_, es posible eliminar algún indicador que no se ajuste adecuadamente con el resto de indicadores de la variable en procura de la aceptación del ajuste global del modelo. En el presente estudio se eliminaron los ítems 9 y 11 por no corresponderse con el modelo global de ajuste.

A continuación se muestra el conjunto de indicadores (denominados "variables observables en un entorno SEM»), donde se puede apreciar que: el análisis del valor tiene seis indicadores: tres de línea base, mientras que el monitoreo, el análisis de costos y la configuración de los productos solo tienen un indicador cada uno. En el rediseño de productos se muestran tres indicadores de los cinco con los que se empezó el análisis, como ya se comentó líneas arriba. En cuanto a la sustitución de componentes, se muestran los tres ítems en el análisis del modelo, los mismos que fueron mantenidos a lo largo del examen.

Seguidamente, se aprecia la tabla 2 de índices de ajuste global incremental y de parsimonia de los modelos de medida y estructura de ecuaciones estructurales con máxima verosimilitud.

Tabla 2. Índices de bondad de ajuste, incrementales y de parsimonia de los modelos de ecuaciones estructurales

\begin{tabular}{l|l|l} 
Índices & Modelo de medida & Modelo de estructura \\
\hline RMSEA & 0.056 & 0.049 \\
\hline PCLOSE & 0.337 & 0.520 \\
\hline CMIN/DF & 1.491 & 1.370 \\
\hline TLI & 0.926 & 0.917 \\
\hline CFI & 0.948 & 0.935 \\
\hline PNFI & 0.613 & 0.634 \\
\hline PCFI & 0.675 & 0.739
\end{tabular}

Fuente: elaboración propia.

La tabla 2 muestra los índices del modelamiento de ecuaciones estructurales con la aceptación de los modelos, con valores en sus índices de ajuste global, incrementales y de parsimonia aceptables. Así, arroja RMSEA menores a 0.08 en ambos casos, PCLOSE mayores a 0.05, CMIN/DF entre 1 y 3 , TLI Y CFI mayores a 0.90, y PNFI y PCFI mayores a 0.50 .

\subsection{Análisis de mediación del rediseño de productos y la sustitución de componentes}

Como segundo objetivo se planteó el estudio del efecto mediador, que podía incluir la sustitución de componentes entre el análisis del valor y el rediseño de productos y la mediación del rediseño de productos entre el análisis del valor y la sustitución de 
componentes. Realizado el análisis de mediación en ambas variables con las macros de Process de Hayes (2017), se observó lo siguiente:

- Del análisis de mediación de la sustitución de componentes entre el análisis del valor y su influencia en el rediseño de productos, se puede comentar que no existen efectos indirectos totales significativos; es decir, no hay mediación de esta variable al obtenerse para la relación un coeficiente de 0.0586 con un nivel de significancia de 0.5257 , superior al 0.05 de aceptación, rechazándose así la hipótesis $\mathrm{H} 4$.

- Todos los efectos directos tienen relaciones significativas, lo que confirma lo encontrado en el análisis de ecuaciones estructurales en sus modelos de medida y de estructura. Esto se puede apreciar en la tabla 3.

- Sobre el análisis de mediación del rediseño entre el análisis del valor y su influencia en la sustitución de componentes, se puede comentar que no existen efectos indirectos totales significativos; es decir, no hay mediación de esta variable al obtenerse para la relación c' un coeficiente de 0.0448 con un nivel de significancia de 0.5257, superior al 0.05 de aceptación, lo que rechaza la hipótesis $\mathrm{H} 3$. Esto se puede observar en la figura 4.

En consecuencia, podemos afirmar que no existe mediación entre las variables análisis del valor y rediseño de productos o sustitución de componentes, respectivamente. Además, se confirma el análisis de modelamiento de medida y de estructura en las ecuaciones estructurales al tener todos los efectos directos coeficientes altos y niveles de significación al 0.000. Esto se puede apreciar en la tabla 3.

Figura 4. Modelo mediación del rediseño y/o la sustitución de componentes

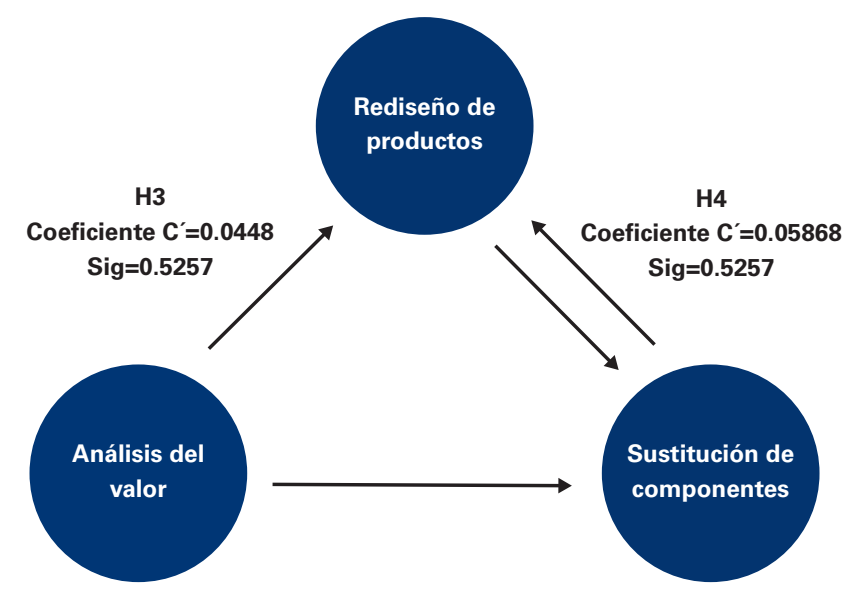

Fuente: elaboración propia. 
La figura 4 nos muestra el modelo de mediación del rediseño de productos, así como de la sustitución de componentes. En ambos casos, se puede apreciar que no existe mediación al tener una c' no significativa.

Tabla 3. Tabla de efectos directos e indirectos de la mediación del análisis del valor con la sustitución de componentes y el rediseño de productos

\begin{tabular}{|c|c|c|c|c|c|}
\hline \multirow[t]{2}{*}{ Coeficientes } & \multirow[t]{2}{*}{ Efectos } & \multicolumn{2}{|c|}{$\begin{array}{l}\text { Mediación sustitución de } \\
\text { componentes }\end{array}$} & \multicolumn{2}{|c|}{$\begin{array}{l}\text { Mediación rediseño de } \\
\text { productos }\end{array}$} \\
\hline & & Coeficientes & Significación & Coeficientes & Significación \\
\hline a & $\begin{array}{l}\text { Efecto directo } \\
\text { (análisis/sustitución) }\end{array}$ & 0.2676 & 0.0000 & 0.5934 & 0.0000 \\
\hline b & $\begin{array}{l}\text { Efecto directo } \\
\text { (sustitución/rediseño ) }\end{array}$ & 0.5770 & 0.0000 & 0.2409 & 0.0000 \\
\hline \multirow[t]{2}{*}{$c^{\prime}$} & $\begin{array}{l}\text { Efecto indirecto } \\
\text { (análisis/sustitución/rediseño) }\end{array}$ & 0.0586 & 0.5257 & 0.0448 & 0.5257 \\
\hline & $\begin{array}{l}\text { Total, efecto indirecto } \\
\text { Límite superior/límite inferior }\end{array}$ & 0.0319 & -0.0416 & 0.0757 & -0.0986 \\
\hline c & $\begin{array}{l}\text { Efecto directo } \\
\text { (análisis/rediseño) }\end{array}$ & 0.5934 & 0.0000 & 0.2675 & 0.0000 \\
\hline
\end{tabular}

Fuente: elaboración propia.

La tabla 3 nos muestra los efectos directos e indirectos del análisis de mediación de sustitución de componentes en la relación entre el análisis del valor y rediseño, observando sus coeficientes y niveles de significación. El análisis de mediación no es significativo, como se muestra en c', y al verificar los límites inferiores y superiores en la mediación de la sustitución de componentes como en la mediación del rediseño de productos, se puede observar que en ambos casos se encuentra el valor "0», lo que confirma la no existencia de mediación.

\subsection{Nivel de utilización del análisis del valor, el rediseño de productos y la sustitución de componentes}

Como tercer objetivo de la investigación se propuso identificar el nivel de utilización de las variables análisis del valor, rediseño de productos y sustitución de componentes por parte de los microempresarios de sector productivo textil en su quehacer diario.

El estudio determinó que el análisis del valor y el rediseño de productos son las variables que registran niveles de utilización altos con $63.10 \%$ y $65.00 \%$, respectivamente. En cuanto a la sustitución de componentes, se obtiene una utilización alta de solo un $37.60 \%$ y una utilización media de 52.90\%; asimismo, esta variable registra el nivel más alto de utilización baja con un $9.60 \%$. El análisis, entonces, determina que los microempresarios de este sector económico si utilizan frecuentemente como base el análisis del valor, el mismo que es usado presumiblemente en la generación de modelos de rediseño de sus productos. En cuanto a la sustitución de componentes, esta es regularmente utilizada. La tabla 4 resume lo comentado. 
Tabla 4. Nivel de utilización de las variables en estudio

\begin{tabular}{l|l|l|l} 
Variables de estudio & Utilización baja & Utilización media & Utilización alta \\
\hline Análisis del valor & $2.50 \%$ & $34.40 \%$ & $63.10 \%$ \\
\hline Rediseño & $3.80 \%$ & $31.20 \%$ & $65.00 \%$ \\
\hline Sustitución de componentes & $9.60 \%$ & $52.90 \%$ & $37.60 \%$
\end{tabular}

Fuente: elaboración propia.

En la tabla 4 se pueden apreciar los niveles de utilización de las variables de estudio, destacando el análisis del valor y el rediseño como aquellas variables que presentan una mayor utilización, así como también un tercio de utilización media, aproximadamente. La sustitución de componentes registra un nivel de utilización media importante, seguida de la utilización alta.

\section{Conclusiones y futuras investigaciones}

\subsection{Conclusiones}

La revisión de la literatura identificó que el análisis del valor se centra fundamentalmente en el análisis de las necesidades de los clientes, de las funciones que son las que dan valor al producto y de los componentes del producto (Garcia Melón et al., 2010), buscando hacer eficiente el manejo de los costos de forma tal que se apliquen las dos alternativas propuestas en la presente investigación: el rediseño y/o la sustitución de componentes.

La investigación planteó como primer objetivo determinar la influencia que tiene el análisis del valor como variable predictora en relación con el rediseño y la sustitución de componentes. De acuerdo a los resultados, se constató en el modelamiento estructural empleado que el análisis del valor influye en relación directa y positiva en el rediseño y en la sustitución de componentes. Sin embargo, se determinó que existe una alta influencia en el caso del rediseño de productos, con un coeficiente de correlación de 0.957 y un nivel de significación de 0.001. En el caso de la sustitución de componentes, existe una correlación de 0.267 y un nivel de significancia de 0.043 , ambos coeficientes dentro del rango de aceptación.

Respecto al segundo objetivo, se precisó que no existe un efecto mediador en ambos casos, pues los resultados indican que no hay un efecto indirecto significativo del rediseño de productos ni de la sustitución de componentes cuando estas están mediando para cada modelo en forma alternada, respectivamente. Además, esto queda demostrado cuando observamos los límites inferiores y superiores, que en ambos casos contienen el valor $« 0$.

El tercer objetivo sobre el nivel de utilización del análisis del valor, rediseño de productos y sustitución de componentes se centra en los ejes centrales para el adecuado manejo de costos en los productos. En este sentido, se determinó que la utilización del análisis del valor y el rediseño de productos son altas (63.10\% y $65 \%$, respectivamente); es más, si se contempla que estas mismas actividades tienen también los niveles medios 
más altos — de $34.40 \%$ y $31.20 \%$, respectivamente—, se puede concluir que son muy empleadas.

En cuanto a la sustitución de componentes, esta actividad tiene un nivel de utilización medio (52.90\%), lo que indicaría que es regularmente aprovechada para reducir costos. Si a ello sumamos su porcentaje de utilización alta, que es de un $37.60 \%$, se concluye que el empleo de esta variable también es importante, aunque tiene una menor incidencia que las dos anteriores. En resumen, estas variables tienen una importante utilización y posiblemente son empleadas como medios para poder alcanzar un incremento de competitividad basado en los costos.

Esta investigación es un aporte al conocimiento disponible, pues a la fecha no se han realizado estudios sobre cómo el análisis del valor influye en el rediseño de productos y en la sustitución de componentes. Actualmente, la gran mayoría de estudios han sido realizados como casos de estudio y, generalmente, el objeto de análisis son las grandes empresas. Es importante comentar que en el análisis del valor se determinan los componentes del producto a manufacturar, la configuración del producto, el costeo del mismo y otros factores que tienen un efecto significativo en la competitividad del negocio, hecho que merece ser investigado.

\subsection{Futuras investigaciones}

La utilización del rediseño y/o sustitución de componentes también se suele realizar sin previo análisis del valor. Esta investigación, no obstante, ha determinado la importancia del análisis del valor, como variable predictor, la misma que tiene una gran influencia en el rediseño de productos y una influencia relevante con la sustitución de componentes. Se espera, pues, que futuras investigaciones confirmen estos hallazgos y puedan ser corroborados en sectores de empresas ensambladoras, donde la sustitución de componentes desempeña un rol más importante que en el sector manufactura.

Asimismo, consideramos que el presente estudio generará interés en seguir investigando temas relacionados al rediseño y la sustitución de componentes como variables mediadoras entre el análisis del valor y la competitividad y/o desempeño organizacional. 


\section{bibliografía}

Abdullah, B., EYT, A., \& Alfadhli, F.

2015

Organización de análisis de valor. Ingeniería de valor (VAVE) durante el desarrollo de nuevos productos (NPD). Revista ARPN de Ingenieria y Ciencias Aplicadas, 10(21), 10052-10057.

\section{Alvarez Suescun, E.}

Decisiones de "hacer o comprar» en el ámbito de los sistemas de información: una aproximación desde la teoría de recursos y capacidades. Cuadernos de Economía y Dirección de Empresa, 10, $223-247$

\section{Bock, S., \& Pütz, M.}

Implementing Value Engineering Based on a Multidimensional Quality-Oriented Control. International Journal of Production Economics, 183, 146-158. doi: http://dx.doi. org/10.1016/j.ijpe.2016.09.007

\section{Carmines, E., \& Zeller, R.}

Reliability and validity assessment. Sage University Papers Series On Quantitative Applications in the Social Sciences, 7(17).

\section{Centro Aragonés de Diseño} Industrial

s.f. Manual para la Gestión del Diseño de Productos en la Empresa. España. Recuperado de: https://www.aragon.es/ documents/20127/674325/07_Manual_G_ Producto\%20Eco.pdf/fcf62c9e-82e3-5c6b965e-c233a8a3039f

Chan-Sik, P., Ho-Jum, K., Hee-Taek, P., Jong-Ho, G., \& Akeem, $P$.
2017 Chen, T., \& Su, T.

Fuzzy-based decision-making applied to performance evaluation in value engineering. Journal of the Chinese Institute of Engineers, 40(3), 200-206.

\section{Chhabra, J., \& Tripathi, J.}

2014

Value Engineering: A Vital Tool for Improving Cost \& Productivity. International Journal of Industrial Engineering \& Technology, 4, 1-10.

De la Gala, B. R., \& Arredondo, $\mathrm{A}$.

La adaptabilidad y alineamiento como variables predictores de la agilidad en las MYPES del sector textil de la región Arequipa, Perú: Un analisis con enfoque triple "A". Espacios, 40(28), 20.

\section{Eckstein, D., Goellner, M.,} Blome, C., \& Henke, M.

2015 The perfomance impact of suply chain agility and suply chain adaptability: The moderating effect of product complexity. International Journal of Production Research, 53(10), 3018 - 3046.

\section{Fornell, C., \& Larcker, D. F.}

Evaluating structural equation models with unobservable variables and measurement error. Journal of Marketing Research, (18), 39-50.

\section{Garcia Melón, M., Alcaide}

Marzal, J., Gómez Navarro, T., Collado Ruiz, D., Peris Blanes,

\section{J., Monterde Diaz, R., y otros}

Fundamentos del Diseño en la Ingeniería. Mexico: Limusa SA. 


\section{bibliografía}

\section{Gonzales, W.}

2007

Las Ciencias de diseño: racionalidad

limitada, predicción y prescripción. España:

Netbiblo.

Guerrer, M., Hernandiz, B., \&

\section{Begoña, V.}

2017 Aproximación a la representación de la forma y apariencia del producto: estudio sobre atributos de diseño. Innovar, 28, 25-39.

Guerrero Valenzuela, M., Hernandis Ortuño, B., \& Agudo Vicent, $B$.

Estudio comparativo de las acciones a considerar en el proceso de diseño conceptual desde la ingeniería y el diseño de productos. Ingeniare. Revista chilena de ingeniería, 22(3), 298-411.

\section{Gupta, U., \& Gupta, A.}

Outsourcing the IS function. Is it necessary for your organization? Information System Management. Information Systems Management, 9(3), 44-50.

\section{Hayes, A.}

Introduction to Mediation Moderation and Conditional Process Analysis A RegresionBased Approach. Guilford.

\section{Houshang, T., Hassan, T., \&} Abdolhossein, $\mathrm{S}$.

2012 El estudio de los factores de organización eficaz en la ejecución de Ingeniería del Valor. Revista Internacional de la Innovación, Gestión y Tecnología, 3(3), 202-205.

\section{Kaveh, M., \& Aminoroaya} Yamini.

2016

The Methodology of Using Value

Engineering in Construction Projects

Management. Civil Engineering Journal,

2(6), 262-269.

\section{Lenau, T., \& Boelskfte, P.}

2004

Soft and Hard Product Atritbutes in Design.

Semantic \& Aestheticc Functions in

Design, 6.

\section{Mana, D.}

2017

Análisis de valor o ongeniería de valor: establecer el nomenclador y el nivel de importancia de las funciones. Revisión de la Dirección General, 26(2), 97-104.

\section{Martínez Argüelles, S., \& Rubiera Morollón, F.}

Hacer versus comprar servicios empresariales. Análisis de las decisiones de externalización de los servicios intensivos en conocimiento en la economia española. Tribuna de Economía, (825), 208-209.

\section{Meeker, D., \& McWilliams, J.}

Structured Cost Reduction Value Engineering by the Numbers. La $18^{a}$ Conferencia internacional anual sobre DFMA Newport. Massachusetts Institute of Technology (MIT). Recuperado de: http:// web.mit.edu/meeker/Public/NE_Triage_ Paper_Final.pdf (consultado el 15 de setiembre de 2018)

\section{Miles, L.}

engineering. 6th Annual Inland Empire Quality Conference. 27 de enero. Recuperado de: https://minds.wisconsin. edu/handle/1793/5616? show=full 


\section{bibliografía}

Morales, $\mathbf{M}$.

2002 ¿Que es ingeniería del valor? Recuperado de: https://minds.wisconsin.edu/

handle/1793/5616?show=full

\section{Polimeri, R.}

1998 Contabilidad de Costos. Concepto y aplicaciones para la toma de decisiones gerenciales. México: McGraw Hill.

\section{Poppo, L., \& Zenger, T.}

1998 Testing alternative theories of the firm: transaction-cost, knowledge-based, and measurement explanations for make-orbuy decisions in information services. Strategic Management Journal, 19, 853 -877. doi: https://doi.org/10.1002/ (SICI)1097-0266(199809) 19:9<853::AIDSMJ977>3.0.CO;2-B

\section{Ramírez Gómez, M.}

2010 Costos de Transaccion y Creación. Revista Ciencias Estratégicas, 18(23), 43-58.

Rui, M., Wenjie, H., Donghao, P., Xiyao, G., Zefeng, L., \& Jie, Z.

2016 Research on lease and sale of electric vehicles. International Journal of Production Research, 54(18), 5361-5380.

\section{Soriau, E.}

Diccionario Akal de estética. Madrid: Akal.

\section{Universidad Interamericana para} el Desarrollo Análisis del producto. Recuperado de: https:// moodle2.unid.edu.mx/dts_cursos_mdl/ADI/ AO/AO01/AOPP01Lectura1.pdf (consultado el 18 de junio de 2019).

Fecha de recepción: 06 de julio de 2018 Fecha de aceptación: 22 de julio de 2018

Sosa Vázquez, R., \& Chávez Alonso, I.

2014 Referentes teóricos a considerar para la toma de decisiones "hacer o comprar" (monografía). Universidad de Matanzas "Camilo Cienfuegos», Cuba. 